\title{
The relevance of spontaneous activity for the coding of the tinnitus sensation
}

\author{
Nathan Weisz ${ }^{1, *}$, Katalin Dohrmann ${ }^{2}$ and Thomas Elbert ${ }^{2}$ \\ ${ }^{1}$ INSERM U821, Brain Dynamics and Cognition, Lyon, France \\ ${ }^{2}$ Universität Konstanz, Konstanz, Germany
}

\begin{abstract}
In this chapter we will present support for the hypothesis that synchronous neuronal activity of cell assemblies within the auditory cortex could be the underlying neural code of tinnitus. Such synchronous activity is reflected in the ongoing oscillatory activation pattern that can be recorded non-invasively using MEG and EEG techniques. We conclude that such an oscillatory model of tinnitus can explain many different observations regarding tinnitus.
\end{abstract}

Keywords: tinnitus; spontaneous activity; oscillations; magnetencephalography

\section{Introduction}

Subjective tinnitus is the sensation of a sound when an identifiable physical source of this perception is absent (see Chapter 1). There is considerable evidence that a lesion in the peripheral auditory system can result in reorganization of central nervous system structures (auditory, but also limbic areas), which may lead to neuronal signals that cause phantom sensations (see Chapters 2 and 3). The most common cause of tinnitus is damage to the receptors of the inner ear. The fact that transection of the auditory nerve does not abolish the tinnitus sensation is strong evidence for the central hypothesis for tinnitus. Animal studies have provided evidence that tinnitus is not associated with hyperactivity of the auditory nerve (Muller et al., 2003), but many studies have found evidence that abnormal (increased) synchrony is involved in tinnitus (see Chapters 2 and 6).

\footnotetext{
*Corresponding author. Tel.: + 314721389 16;

Fax: +31472138901; E-mail: nathanweisz@mac.com
}

This chapter outlines a model of how integrated neuronal activity in the auditory cortex changes following a peripheral lesion that causes tinnitus. In humans with tinnitus this can be best studied using non-invasive electroencephalography (EEG) and magnetencephalography (MEG). The model can be tested and elaborated upon and serve as a base for the treatment of chronic tinnitus. One such clinical approach that has been suggested by the outcome of our recent experimental studies shows that oscillatory brain activity is modified in tinnitus, both in frequency and space (see Chapter 46 for further details).

\section{Deafferentation is essential}

Tinnitus is usually triggered by a dysfunction or lesion in the ear or the auditory nerve. Based on a survey of 5000 tinnitus sufferers, Goebel et al. (2005) found that $80 \%$ report some form of hearing impairment. With these investigators' definition of hearing loss however, apparently a 
considerable proportion of individuals with tinnitus $(20 \%)$ do not have hearing loss and thus have presumably normally functioning hearing. At first glance this supports the view that tinnitus is not necessarily linked to hearing loss and that there may be at least two essentially different forms of tinnitus (e.g., one triggered by peripheral damage and one without any hearing loss). Yet this hypothesis is problematic for two reasons: (1) a transient hearing loss may trigger a reorganization within the auditory system that is maintained even when the hearing function becomes restored. (2) The usual criterion for hearing loss is the clinical audiogram. However, this is a rather incomplete and thus poor assessment of hearing for the following reasons: (1) in almost all individuals with tinnitus whose hearing thresholds according their audiograms were regarded to be within normal limit had abnormal distortion product of otoacoustic emissions (Shiomi et al., 1997) indicating outer hair cell dysfunction. (2) Sharp increased thresholds assessed via the threshold equalizing noise (TEN) test were found in young individuals with tinnitus who had normal (clinical) audiograms, a finding which implies deafferentation (probably inner hair cell damage) in a circumscribed frequency range (Weisz et al., 2006). The TEN test was proposed by Moore et al. (2000) as a screening tool for identifying dead regions on the cochlea. Different from the standard audiogram, the presentation of the pure tone for which the threshold should be assessed is accompanied by a noise that contains equal energy across different auditory filters. When subjects identify the pure tone with functional inner hair cells then thresholds should be close to noise intensity. If the threshold is strongly above $(\sim 10 \mathrm{~dB})$ the noise intensity, then this indicates that the sound is detected by neighboring hair cells. Essentially the TEN is a method to complicate this kind of "offfrequency" listening. (3) Sharp transitions between normal and abnormal hearing — described by the gradient ( $\mathrm{dB} /$ octave) of two neighboring frequencies as it appears in the clinical audiogram - was found to be indicative for the presence of tinnitus by Konig et al. (2006).

All these results point to the involvement of hearing loss or sharp discontinuities in the audiograms in the generation of tinnitus. Deprivation of input likely results in a reduced capacity of affected neurons to inhibit excitatory input from undeafferented neurons located close to the lesion-edge. This in turn is expressed in various changes of central nervous activity of which one (or more) variant could be the neuronal "signature” of tinnitus (Eggermont and Roberts, 2004).

\section{Beyond map reorganization}

One of the effects of a diminished inhibitory capacity is an enlarged representation of sounds in the undamaged regions of the edge of the frequency region that is deprived of input. This means that deprived neurons become responsive to frequencies adjacent to the cortical regions that represent the frequency range where hearing is damaged (so called lesion-edge frequencies). In the somatosensory modality it has been shown that this type of map reorganization is a very fast process, appearing immediately after an amputation of a limb (Calford and Tweedale, 1988). Since morphological reorganization — such as a morphological change of synaptic connectivity with an alteration of synaptic strengths - requires time (at least minutes; Lüscher et al., 2000), the immediateness of the effects has to rely on pre-established connections that are normally silent. On the other hand fast changes (i.e., those realized through migration of receptor proteins into the membrane) usually precede morphological alteration of the synapse (Lüscher et al., 2000). Studies in the chick have shown fast changes in protein synthesis from deprivation of input (Sie and Rubel, 1992). Consequently, if hearing results from loss of hair cells, i.e., a kind of "cochlear amputation" then tinnitus could be the auditory analogue of the somatic phantom pain.

It has been shown in the somatosensory system that the reorganization of the somatotopic map after limb amputation is highly correlated with the amount of reported phantom limb pain (Flor et al., 1995). This would suggest that tinnitus could be related to tonotopic map changes (Mühlnickel et al., 1998). Animal experiments confirm an overrepresentation of lesion-edge frequencies following 
an experimental treatment that would induce tinnitus in humans (Irvine et al., 2001). Moreover, distortions of the tonotopic gradient in subjects with tinnitus have been demonstrated in humans by means of MEG and magnetic source imaging (Weisz et al., 2005b; Wienbruch et al., 2006). It seems important to note that all these observed changes in function were correlational and not causal in nature. An injury, for instance, may trigger another type of plastic alteration in the central auditory system (or beyond) besides map reorganization, and that change may be that what causes tinnitus (see Chapter 3).

Given the network character, it is, however, conceivable that the two processes are somehow linked. Norena and Eggermont (2005) demonstrated in cats that massive high frequency auditory input (enriched acoustic environment) following noise trauma reduced the extent of hearing loss as well as signs of tonotopic map reorganization in the primary auditory cortex. It will be of interest to see if such stimulation affects tinnitus in humans.

In addition, there is a central reorganization in neural networks that is probably not directly linked to map reorganization and that is expressed in characteristic alterations of ongoing spontaneous activity extending much beyond the location of the altered tonotopic map (Weisz et al., 2005a). It remains to be tested how closely the two phenomena are linked to each other. In the following, we will present the hypothesis that tinnitus is more directly related to the changes in the responses pattern of neurons, especially their oscillatory behavior than to map reorganization, and that retuning of the former modifies tinnitus (see Dohrmann et al., this volume). Additionally, the same enriched acoustic environment mentioned previously also prevents changes of spontaneous activity putatively linked to tinnitus (Norena and Eggermont, 2006).

\section{Tinnitus and variants of spontaneous activity}

As the title of this chapter suggests, spontaneous activity is a rather generic term used here to address any neuronal activity that is not evoked or driven by an external event. This is an important point as this definition overlaps with that of the definition of tinnitus. The term spontaneous activity can be a constant source of confusion, as it may describe features on various scales - from firing of single units to oscillatory activity of large cell assemblies - which strongly depends on the background of the researcher and the individual with tinnitus (human, animal) who is investigated. We will first give a short description of findings from animal research, which usually focuses on spontaneous activity of a single neuron or a few neurons. Subsequently, a brief introduction to oscillatory brain responses will be given. Oscillatory responses can be studied in humans using noninvasive techniques. We will show that this is also the level of neuronal activity that is likely to be related to higher order cognitive functions, such as perception and attention.

\section{Insights from animal studies}

As mentioned above one of the basic assumptions regarding tinnitus is its relationship to a reduced inhibitory capacity of neurons that are deprived of input. This is supposed to lead to a hyperactivation of neighboring neurons that are not deprived of input characterized by increased neuronal firing rate. The only level of the auditory nervous system where this has been consistently shown appears to be the dorsal cochlear nucleus (Kaltenbach, 2006). For the inferior colliculus, reported results are inconsistent. While Chen and Jastreboff (1995) find increases in firing rate following administration of salicylate to induce tinnitus, significant decreases relative to baseline were reported by Ma et al. (2006) using doses of salicylate proven to be sufficient to induce tinnitus. Yet spontaneous firing rates increased after exposure to noise that was assumed to have induced tinnitus as reported by $\mathrm{Ma}$ et al. Since both exposure to noise and the administration of salicylate were expected to have caused tinnitus enhanced firing of neurons in the inferior colliculus does not appear to be a consistent neuronal correlate of phantom sound perception. Results from similar studies of the auditory cortex are not conclusive either, with 
varying results depending on the way tinnitus is induced. Increased firing was noticed by Norena and Eggermont (2003) in the primary auditory cortex following noise trauma, but a reduction is seen when tinnitus is elicited by salicylate (Eggermont and Kenmochi, 1998). Increases in firing rate of neurons in the secondary auditory cortex following salicylate and quinine application have been reported (Eggermont and Kenmochi, 1998).

Interpretation of results from animal experiments are hampered by the anesthesia used which may affect the results in unknown ways. Yang et al. (2006) pointed out that after administration of anesthesia the mean spontaneous firing rate (even in studies reporting hyperactivity) was still on the order of 7-11 times lower than in nonanesthetized animals. For awake rats these authors found that administration of salicylate reduced spontaneous firing rate (hypoactivity). These results that should exemplify that a pure hyperactivity logic appears to be insufficient is furthermore corroborated by the temporal evolution of these spontaneous rate effects (Eggermont and Roberts, 2004): after intense noise exposure enhancements of firing rate in the primary auditory cortex develop after a few hours (Norena and Eggermont, 2003) and even 2-5 days in the dorsal cochlear nucleus (Kaltenbach et al., 2000). This contrasts with the normally rapid onset of tinnitus following noise trauma.

Given these findings, it is likely that the mechanisms causing the tinnitus are likely to go beyond a pure quantitative excess of activity in the form of an increased neuronal firing rate. An alternative would be that distinct populations of neurons within the auditory cortex coding the phantom percept synchronize their firing. Such synchronization may accompany an overall increase in firing, but not necessarily be tied to that (see also Chapter 2). Summation of post-synaptic potentials induced by synchronized input is stronger than that from asynchronous spikes, thus prioritizing features that are coded via synchrony for further processing (Niebur et al., 2002). Spike synchronization has been particularly well-studied in the visual modality, where it has been associated with representation of stimulus features within a cell assembly and binding of features across distributed assemblies into conscious percepts (see Singer, 1999 for an extensive review).

Far less is known about the role of such synchronization in the auditory system; yet it is imaginable that the tinnitus percept could arise through "intrinsically" generated synchrony, i.e., unrelated to an external presentation of an auditory stimulus. The ensemble firing created would then attain more saliency than dispersed firing and be interpreted as real sound at subsequent processing stages. Prolonged synchronous firing then will lead to use-dependent synaptic modifications (long-term potentiation, LTP), creating a stabilized tinnitus related cell assembly with time. Indeed there is some evidence that this might occur: Synchronous spiking (normalized for the total number of spikes) has been shown to increase in the primary auditory cortex of cats following noise trauma (Norena and Eggermont, 2003; Seki and Eggermont, 2003). Importantly, the change to synchronized firing appears to occur more rapidly than changes in firing rate, (Norena and Eggermont, 2003; Seki and Eggermont, 2003) after noise trauma. These findings have been obtained in animals being under anesthesia; albeit under a state where it seems even more challenging than normal to know whether they may be accompanied by correlates on a perceptual level. There is therefore a need to study synchrony in unanaesthetized animals and such studies should be complemented by electrophysiological studies in humans.

\section{Work in humans: oscillations reflect integrated activity from neuronal assemblies}

Intracranial recordings of spike activity of single or multiple units in humans is possible only under exceptional circumstances, often limited to pathological conditions of one kind or another. To the best of our knowledge there have been no reports to date regarding local field potentials (LFPs) from the auditory cortex in humans with tinnitus. Noninvasively, spontaneous activity can be recorded by means of EEG or MEG. It is important to stress once again that this is not identical to what is commonly meant by spontaneous activity in 
animal studies, which refers to firing properties in general and not necessarily to synchrony. EEG and MEG on the other hand represent the ensemble electrical activity (i.e., from their post-synaptic potentials) of large neuronal populations or cell assemblies. Apart from being non-invasive, these methods have the distinct advantage of giving access to assembly activity as it unfolds in real time (only limited by the sampling rate). A disadvantage is that simultaneous activities overlap and, consequently, the anatomical location of the activity that is recorded from the scalp cannot be inferred directly from the data (or sometimes not at all). The anatomical location of the sources can only be approximated via inverse solution strategies that rely on certain assumptions.

Oscillatory activity has been and still is frequently associated with different cognitive or pathological states. Since the EEG/MEG signals represent the combination of excitatory and inhibitory post-synaptic potentials, oscillatory activity can be regarded as an integration of input. Analysis, such as frequency (spectrum) analysis or autocorrelation analysis, can provide quantitative information about oscillatory components of recorded electrical potentials such as EEG. Wavelet analysis can provide time-frequency representation relative to an external or internal event. Furthermore, changes in the post-synaptic potentials alter the probability of spikes (i.e., output), so oscillatory activity also reflects fluctuations in neuronal excitability. It has been shown, for example, that spike synchronization is accompanied by rhythmic firing in the gamma range, itself closely related to the oscillatory response of the LFP (Gray and Singer, 1989). Also, spike-triggered averaging has revealed concomitant gamma activity in the LFP (Fries et al., 2001). Yet, spiking and gamma activity appears also to be modulated by slower frequency oscillations (Lakatos et al., 2005; Lee et al., 2005). It is one of the fascinating aspects of brain function that oscillatory activity is self-organizing, i.e., under certain circumstances activation properties of single cells turn into population properties via synchronization. The integrated activity leads to behaviors, sensations, feelings, etc., putting oscillatory brain activity at the interface of the mind-body problem.

\section{Tinnitus and ongoing oscillatory activity in humans}

A large amount of data has been acquired from studies of animal models of tinnitus. Only very little is known about the relationship of ongoing spontaneous activity and tinnitus in humans despite the known behavioral importance of largescale oscillatory activity. The great majority of electrophysiological studies in humans with tinnitus are still guided by an event-related approach, i.e., by averaging signals obtained after presenting an event. In a series of studies, our group has focused on spontaneous neuronal activity. We have specifically studied the involvement of different frequency bands in the generation and maintenance of tinnitus and the results serve as elements of a neural oscillation model of tinnitus. The results will be summarized below. We will not cover event related/driven oscillatory activity such as steady-state responses, which is also an active area of research in our group (Schlee et al., 2007; Wienbruch et al., 2006).

In one approach (Weisz et al., 2005a), we assessed $5 \mathrm{~min}$ of resting MEG activity in patients with tinnitus and compared the power spectra to those of normal hearing controls. The main differences were markedly reduced alpha power $(8-12 \mathrm{~Hz})$ and an increased low-frequency power (delta; $1-4 \mathrm{~Hz}$ ). These differences were most pronounced bilaterally over perisylvian areas, thus potentially stemming from auditory cortex. The abnormalities in the activity recorded over frontotemporal regions were significantly correlated to tinnitus-related distress. This finding leads to the hypothesis that tinnitus encompasses a distributed network of neurons in different brain regions including auditory and non-auditory areas that process basic (phantom) sound sensation and related affective and motivational aspects respectively (see also Schlee et al., 2007).

Other parts of our recent research concern how the observed abnormal spontaneous activity pattern is related to perception of sound. As mentioned above there are reasons to believe that synchronous neuronal activity in the gamma band could underlie conscious perception of sound, which includes tinnitus. In a recent study (Weisz et al., 2007), we focused on the high frequency 
oscillatory dynamics in patients with tinnitus and normal-hearing controls particularly during periods of enhanced slow-wave activity: pronounced peaks in the $2-7 \mathrm{~Hz}$ band-pass filtered and Hilberttransformed data were used to identify these periods. This work yielded three important findings: (1) the time-course of the slow-wave activity was strongly correlated to activity in a frequency band between 50 and $60 \mathrm{~Hz}$, particularly in controls. (2) Overall, activity in the gamma band was increased in individuals with tinnitus. (3) Activity $\sim 55 \mathrm{~Hz}$ was significantly associated to the laterality of the tinnitus percept. This is the frequency range $(50-60 \mathrm{~Hz})$ that was modulated by slow-wave activity in controls, implicating in general a coupling between these bands. Individuals with unilateral or unilaterally dominant tinnitus had stronger $55 \mathrm{~Hz}$ activity contralateral to the reported sensation, whereas subjects with equally strong tinnitus on both sides showed no such lateralization. We interpreted these finding to indicate that the enhanced gamma activity reflect the synchronous firing of neurons within the auditory cortex. This means that the observed gamma activity could be the neurophysiological correlate of basic sound perception. We will elaborate on that below and integrate the interpretations of the different findings.

A single measurement of resting spontaneous EEG/MEG activity does not answer the question about how abnormalities in ongoing oscillatory activity may be related to tinnitus. Attempts to modify ongoing spontaneous activity and measure changes in the perception or, manipulate the tinnitus and observe concomitant changes in brain activity are methods that are likely to contribute to understanding of how the electrophysiological abnormalities in tinnitus patients are related to the tinnitus and its character. The first strategy is currently being tested using neuro-feedback in which the aim is to normalize the spontaneous activity pattern by enhancing alpha power and reducing delta power. Preliminary results indicate that concomitant changes in both bands lead to the greatest reductions of tinnitus loudness (for detailed information see Chapter 46). The other strategy consists of reducing the intensity of the tinnitus and to observe whether concomitant changes occur in the electrophysiological activity. One way to achieve changes in loudness is by using the phenomenon of residual-inhibition (RI) (see Chapter 47), which is a transient reduction in the tinnitus intensity (or even an abolished sensation) that outlasts the duration of a masking sound. We have tested the effect of an RI sound as compared to a control sound on spontaneous activity in eight individuals with tinnitus (Weisz et al., 2007). The most pronounced effect was a significantly reduced delta activity for the RI sound only (see Fig. 1). Overall, the behavioral effects, however, were rather weak but we would expect larger effects in connections with greater reduction of tinnitus through RI. Nevertheless, this study, as well as the others presented here, indicates that some forms of tinnitus may be caused by abnormal spontaneous activity pattern in ensembles of neurons, and that it could be possible to achieve significant relief by normalizing this pattern of neural activity.

\section{Concluding remarks and a model proposal}

In this chapter we have elaborated on the hypothesis that tinnitus may be the consequence of an enhanced level of synchronous firing of neurons in the auditory cortex in absence of any external activation. On a more macroscopic level these changes are associated with an altered pattern of ongoing oscillatory activity as can be measured non-invasively in humans using EEG and MEG. We are not aware of any studies reporting spontaneous LFP activity in awake animals following tinnitus induction. It would be of great interest and importance to relate spontaneous activity LFP data in animals to human EEG/MEG data and vice versa. ${ }^{1}$ Certainly focusing only on a cortical level constitutes an oversimplification as abnormalities can be also observed at subcortical levels

\footnotetext{
${ }^{1}$ Even though neuroimaging data (PET, fMRI) can contribute some interesting aspects in interpreting electrophysiological data it is necessary not to confuse the methods. Neuroimaging methods essentially measure changes in metabolism and cerebral blood flow and the relationship with neuronal activity is by no means clear. There is some evidence that the BOLD response reflects local field potentials and particularly gamma activity (Logothetis, 2002), yet more recent studies cast doubts that there is a direct relationship (Burke and Buhrle, 2006).
} 
A)

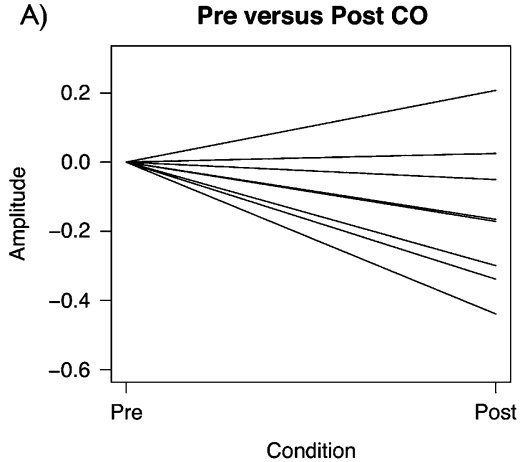

B)

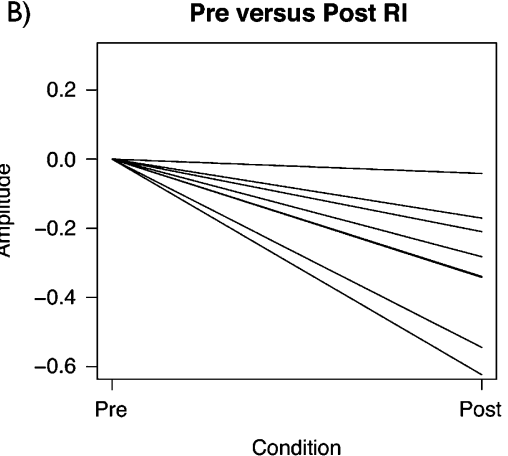

Fig. 1. Delta activity for a period pre and post presentation of two masking stimuli. One stimulus (RI) induced a more pronounced residual inhibition than the other (control, CO). Each subject is depicted as a line and the pre value was set to zero in order to better visualize the changes. The RI sound reduced slow-wave activity in every single subject, whereas a greater variability was present for the CO stimulus.

in animal models of tinnitus. While it may seem logical to assume that the lowest level in the auditory system showing abnormal neuronal activity must be the place of origin of the tinnitus sensation, this hypothesis is flawed for several reasons. The auditory system comprises feed-forward and feedback circuits that cause complex interactions between different parts of the system. The fact that the fibers from the auditory cortex to the medial geniculate body outnumber the thalamocortical ones by a factor ten (Eggermont, 2003) is a strong sign of such interaction and indicates that spontaneous activity (as well as tonotopic maps) can be altered in a descending manner as well (Suga and Ma, 2003).

This leads to our first conclusion that an altered pattern of ongoing oscillatory activity in the auditory cortex is the underlying neural code of tinnitus. In particular, enhancements in the gamma frequency band, which can be assumed to be a sign of enhanced synchronized firing of neurons, appear to be involved in the formation of a conscious percept of phantom perceptions such as tinnitus. How are changes in neural activity reflected in other frequency bands of the EEG? Slow-wave activity may reflect so-called low-threshold spike (LTS) bursts generated in thalamic nuclei as a consequence of hyperpolarization (Llinas et al., 1999; Jeanmonod et al., 1996). However, the relation of such activity to tinnitus is unknown. On the other hand, it is well known that the cortical architecture is rich in inhibitory interneurons (InhIn) that enable rapid modification of cortical neuronal activity. Bursting activity is also not normally evident in the auditory cortex (Eggermont and Roberts, 2004) or only transiently following noise trauma (Norena and Eggermont, 2003). Furthermore, slower synaptic changes (perhaps implied in stabilizing the "tinnitus cell-assembly") mainly affect intracortical excitatory synapses (layer 2/3), rather than thalamocortical synapses (layer 4; Foeller and Feldman, 2004). For these reasons, our oscillatory model assumes that the relevant processes leading to the phantom sound take place on a cortical level. This model is an extension of the model proposed by Eggermont (Eggermont and Roberts, 2004) in which we have incorporated oscillatory brain activity.

First, we assume that damage to the cochlea essentially leads to a deprivation of neurons tuned to frequencies that correspond to the frequencies where the hearing threshold is elevated, while sparing neurons that are tuned to other frequencies (indicated by the arrow thickness in Fig. 2). Neurons in the nuclei of the ascending auditory pathways including the cerebral cortex are arranged tonotopically and ultimately form the cortical representational map. In a simplified description, the auditory cortex may be regarded as consisting of a layer of excitatory pyramidal neurons (ExPy) and InhIn. These neurons interact via inhibitory or excitatory intracortical synapses 


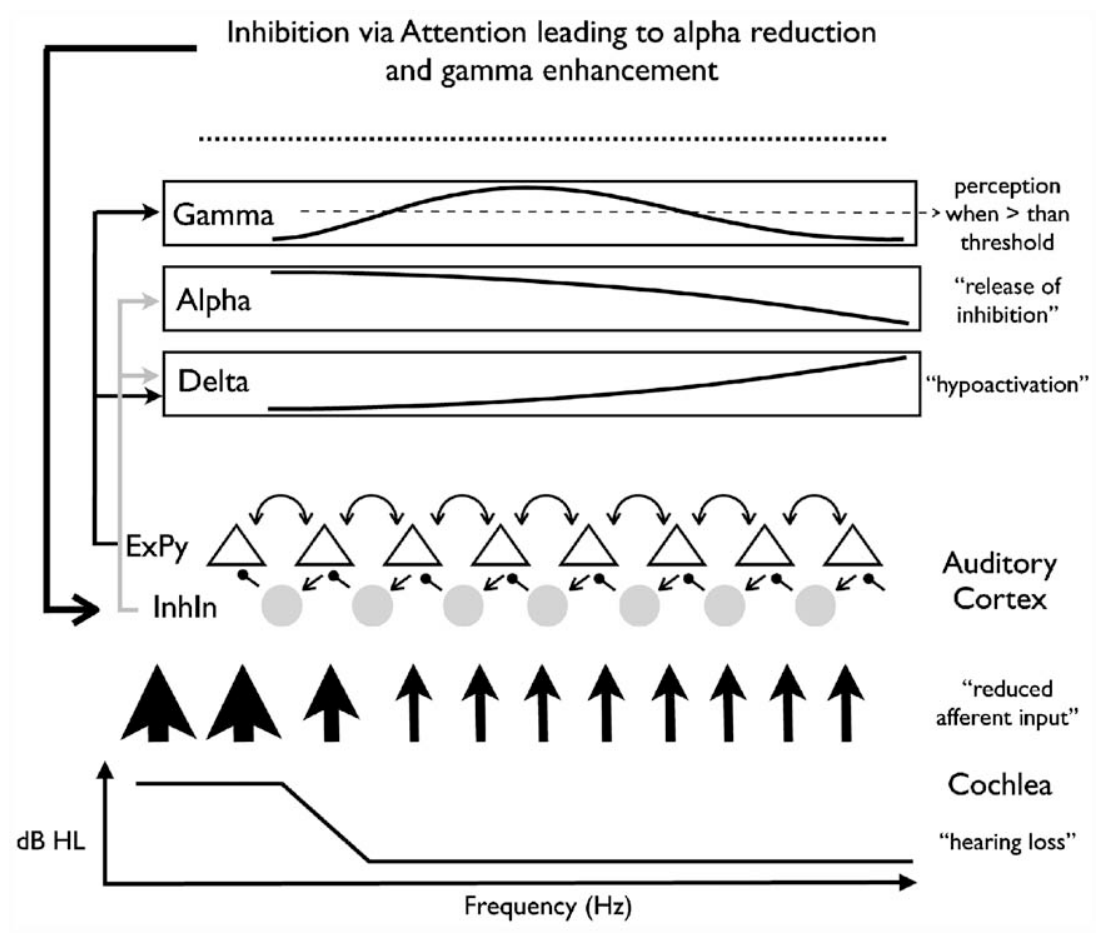

Fig. 2. Oscillatory model of tinnitus. Input deprivation is indicated by an increased threshold in $\mathrm{dB} H \mathrm{HL}$ and reduced line thickness. This leads to reduced activity ("hypoactivity"), marked by an increasing delta activity along the tonotopic axis. A reduced activation of inhibitory neuronal units changes the normal synchronization frequency, which is hypothesized to be in the alpha range (see decreasing alpha slope along tonotopic axis). At a spatial location, where firing probability of excitatory pyramidal neurons is increased via a "release of inhibition" and at the same time affected minimally by hypoactivation (i.e., in principle still responsive), a circumscribed region of gamma activity should emerge. This high-frequency synchronization of neuronal activity putatively underlies the sound perception. The activational pattern can furthermore be modulated attention, which is hypothesized to act mainly on the inhibitory neurons. See text for a detailed explanation. ExPy, excitatory pyramidal neurons; InhIn, inhibitory interneurons.

(for simplicity the inhibitory connections to excitatory neurons with higher characteristic frequencies (CFs) are omitted as are the excitatory connections between neurons with lower $\mathrm{CF}$ and the inhibitory neuron).

A critical assumption of this model is that under a condition of no external input the normal synchronizing activity of InhIn will be in the alpha range. This is in accordance with the framework of Miller, (2006) who assumed that: (1) EEG alpha activity reflects a hyperpolarized ("down") state of a neuronal assembly, the positive peaks of which coincide with the bursting activity that can be recorded intracranially. (2) Bursting activity (interspike intervals $<10 \mathrm{~ms}$ ) itself makes activation of InhIn via pyramidal cells more likely, keeping the neuronal assembly in a "down" state. However, a deafferentiation would modify the balance of interactions, possibly resulting in a massive change of the set of attractors in this non-linear system. Reduced afferent input reduces the spontaneous firing of inhibitory neurons (marked by decreasing alpha-line in Fig. 2). This condition should normally lead to increased neuronal activity with synchronization in the gamma frequency range ("release of inhibition"). However, this augmenting tendency is counteracted by a reducing tendency, as the deafferentiation not only hyperpolarizes the InhIn but also the ExPy. This is shown as an increasing "hypoactivation" line in Fig. 2. Due to the excitatory inputs of neurons at the audiometric edge, the increasing hypoactivation or release of inhibition are supposed to be smooth. In a certain region of the tonotopic map, 
an increase of synchronized neuronal activity will emerge where these two opposing tendencies cross. This represents a region where a release of inhibition is strong enough to enhance activity of the ExPy and the hypoactivation that occurs at the same time is not strong enough to make firing of ExPy improbable. In our view, the locally accentuated gamma activation constitutes the neuronal correlate of the tinnitus perception.

This model is able to explain several empirical findings and observations: The model predicts that the enhanced gamma activity is not located at the audiometric edge but within the frequency range of hearing loss, as reported by Norena and Eggermont (2003). The model also explains why only parts of the region that is deprived of input synchronize, which is in keeping with the observation that the majority of the participants in tinnitus studies report a tonal sensation (i.e., they say that their perception has a distinct pitch even though they may have problems when it comes to matching the frequency of their tinnitus to a tone). Finally, the model emphasizes the importance of topdown mechanisms, which influence the subjective loudness of tinnitus by modulating neuronal activity in the auditory cortex through higher structures such as emotion and attention controlling systems. The model assumes that the descending (top-down) influence is exerted largely via inhibitory connections to InhIn. Periods of focused attention toward the tinnitus sound lead to a further alpha desynchronization and gamma synchronization on an electrophysiological level (Bauer et al., 2006). The overall spectral changes associated with tinnitus become more pronounced aggravating the distress on a subjective and behavioral level.

Certainly this model needs to be refined. Yet it creates some testable hypotheses that we hope will lead to further neuroscience research of tinnitus.

\section{Abbreviations}

$\begin{array}{ll}\text { CF } & \text { characteristic frequency } \\ \text { EEG } & \text { electroencephalography } \\ \text { ExPy } & \text { excitatory pyramidal neurons } \\ \text { InhIn } & \text { inhibitory interneurons } \\ \text { LFP } & \text { local field potential }\end{array}$
LTP
LTS
MEG
RI
long-term potentiation
low-threshold spike
magnetencephalography
residual-inhibition
TEN

\section{Acknowledgments}

Our work on tinnitus is funded by the Deutsche Forschungsgemeinschaft and the Tinnitus Research Initiative. We thank Winfried Schlee and Olivier Bertrand for useful discussions that helped to form the ideas formulated here.

\section{References}

Bauer, M., Oostenveld, R., Peeters, M. and Fries, P. (2006) Tactile spatial attention enhances gamma-band activity in somatosensory cortex and reduces low-frequency activity in parieto-occipital areas. J. Neurosci., 26: 490-501.

Burke, M. and Buhrle, C. (2006) BOLD response during uncoupling of neuronal activity and CBF. NeuroImage, 32: 1-8.

Calford, M.B. and Tweedale, R. (1988) Immediate and chronic changes in responses of somatosensory cortex in adult flyingfox after digit amputation. Nature, 332: 446-448.

Chen, G.D. and Jastreboff, P.J. (1995) Salicylate-induced abnormal activity in the inferior colliculus of rats. Hear. Res., 82: 158-178.

Eggermont, J.J. (2003) Central tinnitus. Auris Nasus Larynx, 30(Suppl): S7-S12.

Eggermont, J.J. and Kenmochi, M. (1998) Salicylate and quinine selectively increase spontaneous firing rates in secondary auditory cortex. Hear. Res., 117: 149-160.

Eggermont, J.J. and Roberts, L.E. (2004) The neuroscience of tinnitus. Trends Neurosci., 27: 676-682.

Flor, H., Elbert, T., Knecht, S., Wienbruch, C., Pantev, C., Birbaumer, N., Larbig, W. and Taub, E. (1995) Phantomlimb pain as a perceptual correlate of cortical reorganization following arm amputation. Nature, 375: 482-484.

Foeller, E. and Feldman, D.E. (2004) Synaptic basis for developmental plasticity in somatosensory cortex. Curr. Opin. Neurobiol., 14: 89-95.

Fries, P., Reynolds, J.H., Rorie, A.E. and Desimone, R. (2001) Modulation of oscillatory neuronal synchronization by selective visual attention. Science, 291: 1560-1563.

Goebel, G., Knoer, H., Knoer, E. and Hiller, W. (2005) Profile of the German Tinnitus League (DTL): Characteristics of the Members Compared with Epidemiological Dates. In: Daumann, R. and Bouscall-Faure, F. (Eds.), Proceedings of the VII th International Tinnitus Seminar:182.

Gray, C.M. and Singer, W. (1989) Stimulus-specific neuronal oscillations in orientation columns of cat visual cortex. Proc. Natl. Acad. Sci. U.S.A., 86: 1698-1702. 
Irvine, D.R., Rajan, R. and Brown, M. (2001) Injury- and use-related plasticity in adult auditory cortex. Audiol. Neurootol., 6: 192-195.

Jeanmonod, D., Magnin, M. and Morel, A. (1996) Low-threshold calcium spike bursts in the human thalamus: common physiopathology for sensory, motor and limbic positive symptoms. Brain, 119: 363-375.

Kaltenbach, J.A. (2006) Summary of evidence pointing to a role of the dorsal cochlear nucleus in the etiology of tinnitus. Acta Otolaryngol. Suppl., 556: 20-26.

Kaltenbach, J.A., Zhang, J. and Afman, C.E. (2000) Plasticity of spontaneous neural activity in the dorsal cochlear nucleus after intense sound exposure. Hear. Res., 147: 282-292.

Konig, O., Schaette, R., Kempter, R. and Gross, M. (2006) Course of hearing loss and occurrence of tinnitus. Hear. Res. 221: 59-64.

Lakatos, P., Shah, A.S., Knuth, K.H., Ulbert, I., Karmos, G. and Schroeder, C.E. (2005) An oscillatory hierarchy controlling neuronal excitability and stimulus processing in the auditory cortex. J. Neurophysiol., 94: 1904-1911.

Lee, H., Simpson, G.V., Logothetis, N.K. and Rainer, G. (2005) Phase locking of single neuron activity to theta oscillations during working memory in monkey extrastriate visual cortex. Neuron, 45: 147-156.

Llinas, R.R., Ribary, U., Jeanmonod, D., Kronberg, E. and Mitra, P.P. (1999) Thalamocortical dysrhythmia: a neurological and neuropsychiatric syndrome characterized by magnetoencephalography. Proc. Natl. Acad. Sci. U.S.A., 96: $15222-15227$.

Logothetis, N.K. (2002) The neural basis of the blood-oxygenlevel-dependent functional magnetic resonance imaging signal. Philos. Trans. R. Soc. Lond. B Biol. Sci., 357: 1003-1037.

Lüscher, C., Nicoll, R.A., Malenka, R.C. and Muller, D. (2000) Synaptic plasticity and dynamic modulation of the postsynaptic membrane. Nature Neuroscience, 3: 545-555.

Ma, W.L., Hidaka, H. and May, B.J. (2006) Spontaneous activity in the inferior colliculus of CBA/J mice after manipulations that induce tinnitus. Hear. Res., 212: 9-21.

Miller, R. (2006) Theory of the normal waking EEG: from single neurones to waveforms in the alpha, beta and gamma frequency ranges. Int. J. Psychophysiol., 64: 18-23.

Moore, B., Huss, M., Vickers, D., Glasberg, B. and Alcantara, J. (2000) A test for the diagnosis of dead regions in the cochlea. Br. J. Audiol., 34: 205-224.

Muller, M., Klinke, R., Arnold, W. and Oestreicher, E. (2003) Auditory nerve fibre responses to salicylate revisited. Hear. Res., 183: 37-43.

Mühlnickel, W., Elbert, T., Taub, E. and Flor, H. (1998) Reorganization of auditory cortex in tinnitus. Proc. Natl. Acad. Sci. U.S.A., 95: 10340-10343.

Niebur, E., Hsiao, S.S. and Johnson, K.O. (2002) Synchrony: a neuronal mechanism for attentional selection? Curr. Opin. Neurobiol., 12: 190-194.
Norena, A.J. and Eggermont, J.J. (2003) Changes in spontaneous neural activity immediately after an acoustic trauma: implications for neural correlates of tinnitus. Hear. Res., 183: 137-153.

Norena, A.J. and Eggermont, J.J. (2005) Enriched acoustic environment after noise trauma reduces hearing loss and prevents cortical map reorganization. J. Neurosci., 25: 699-705.

Norena, A.J. and Eggermont, J.J. (2006) Enriched acoustic environment after noise trauma abolishes neural signs of tinnitus. Neuroreport, 17: 559-563.

Schlee, W., Weisz, N., Dohrmann, K., Hartmann, T. and Elbert, T. (2007) Scrutinizing the tinnitus-distress network using single trial auditory steady state responses. In: Cheyne D., Ross B. and Weinberg H. (Eds.), Proceedings of the 15th International Conference on Biomagnetism, Elsevier, Amsterdam.

Seki, S. and Eggermont, J.J. (2003) Changes in spontaneous firing rate and neural synchrony in cat primary auditory cortex after localized tone-induced hearing loss. Hear. Res., 180: 28-38.

Shiomi, Y., Tsuji, J., Naito, Y., Fujiki, N. and Yamamoto, N. (1997) Characteristics of DPOAE audiogram in tinnitus patients. Hear. Res., 108: 83-88.

Sie, K.C.Y. and Rubel, E.W. (1992) Rapid changes in protein synthesis and cell size in the cochlear nucleus following eighth nerve activity blockade and cochlea ablation. J. Comp. Neurol., 320: 501-508.

Singer, W. (1999) Neuronal synchrony: a versatile code for the definition of relations? Neuron, 24: 111-125.

Suga, N. and Ma, X. (2003) Multiparametric corticofugal modulation and plasticity in the auditory system. Nat. Rev., 4: 783-794.

Weisz, N., Hartmann, T., Dohrmann, K., Schlee, W. and Norena, A. (2006) High-frequency tinnitus without hearing loss does not mean absence of deafferentation. Hear. Res., 222: $108-114$

Weisz, N., Moratti, S., Meinzer, M., Dohrmann, K. and Elbert, T. (2005a) Tinnitus perception and distress is related to abnormal spontaneous brain activity as measured by magnetoencephalography. PLoS Med., 2: e153.

Weisz, N., Müller, S., Schlee, W., Dohrmann, K., Hartmann, T. and Elbert, T. (2007) The neural code of auditory phantom perception. J. Neurosci., 27(11): 1479-1484.

Weisz, N., Wienbruch, C., Dohrmann, K. and Elbert, T. (2005b) Neuromagnetic indicators of auditory cortical reorganization of tinnitus. Brain, 128: 2722-2731.

Wienbruch, C., Paul, I., Weisz, N., Elbert, T. and Roberts, L.E. (2006) Frequency organization of the $40 \mathrm{~Hz}$ auditory steadystate response in normal hearing and in tinnitus. NeuroImage, 33: 180-194.

Yang, G., Lobarinas, E., Zhang, L., Turner, J., Stolzberg, D., Salvi, R. and Sun, W. (2006) Salicylate induced tinnitus: behavioral measures and neural activity in auditory cortex of awake rats. Hear. Res., 226: 244-253. 